Cahiers $d u$ MONDE RUSSE

\section{Cahiers du monde russe}

Russie - Empire russe - Union soviétique et États indépendants

$60 / 4 \mid 2019$

Varia

\title{
Ilya GERASIMOV, Plebeian Modernity. Social practices, Illegality, and the Urban Poor in Russia, 1906-1916
}

Dorena Caroli

\section{(2) OpenEdition}

\section{Journals}

Édition électronique

URL : https://journals.openedition.org/monderusse/11498

DOI : $10.4000 /$ monderusse. 11498

ISSN : $1777-5388$

Éditeur

Éditions de l'EHESS

Édition imprimée

Date de publication : 1 octobre 2019

Pagination : 830-833

ISBN : 978-2-7132-2797-4

ISSN : $1252-6576$

Référence électronique

Dorena Caroli, « Ilya GERASIMOV, Plebeian Modernity. Social practices, Illegality, and the Urban Poor in Russia, 1906-1916 », Cahiers du monde russe [En ligne], 60/4 | 2019, mis en ligne le 01 octobre 2019, consulté le 06 janvier 2023. URL : http://journals.openedition.org/monderusse/11498 ; DOI : https:// doi.org/10.4000/monderusse. 11498 
Ilya GERASIMOV

\section{Plebeian Modernity}

Social practices, Illegality, and the Urban Poor in Russia, 1906-1916

Rochester : University of Rochester Press, 2018, ix +275 p.

Cet ouvrage d'Ilya Gerasimov est issu d'une recherche de longue haleine sur l'histoire du postnationalisme moderne et sur l'histoire sociale de la Russie impériale, menée par son auteur parallèlement à son activité de codirecteur de la prestigieuse revue Ab Imperio. Il traite de l'histoire de groupes sociaux très particuliers de quatre importantes villes russes (Vil'na, Odessa, Kazan' et Nižnij-Novgorod) de 1906 à 1916. Ces villes sont dépeintes d'un point de vue démographique, économique et social. Dans le contexte de ces années 1906-1916, la majorité de la population, constituée d'émigrés des campagnes, illettrés et étrangers aux discours de l'élite, représentait une catégorie sociale particulière de la ville tsariste. C'est dans des sources très variées, mais surtout criminelles, que l'auteur poursuit sa recherche sur ces individus ordinaires, dans le but de renouveler les études fondées souvent sur des sources savantes ayant contribué à créer des stéréotypes liés principalement aux caractères ethniques. 
Cet ouvrage est novateur : il est le premier à aborder la question des conduites illégales des couches populaires par le biais de leurs pratiques sociales considérées en tant que traces non verbales de leurs actions et réactions violentes, dans un milieu urbain multiethnique marqué par des courants plus ou moins forts de modernisation et par des institutions gouvernant et contrôlant la population urbaine de façon très diverse. L'ouvrage, précédé d'une introduction intitulée « Les subalternes parlent fort : Gerasim et les infâmes », comporte cinq chapitres, un épilogue (avec des notes en fin de volume), une bibliographie sélective et un index des noms.

Dès l'introduction, l'auteur annonce sa démarche, une démarche très originale : Gerasim, le personnage principal du récit de Turgenev Mumu, un paysan sourd-muet devenu domestique, devient un véritable fil rouge, symbole de l'oppression de la population russe subalterne, de la rébellion et du changement individuel et collectif. Du point de vue méthodologique, le livre se situe dans un cadre complexe d'études sur le concept de subalternité en Russie, inspiré de Habermas et de Foucault ; il cherche à élaborer une méthode nouvelle, mais il a recours aussi aux méthodes de l'anthropologie culturelle pour saisir le sens des pratiques sociales. Cependant, l'auteur s'éloigne de l'analyse des discours, car il se situe aussi dans l'histoire de l'urbanisme impérial, en renouvelant considérablement les connaissances sur le tissu social des quatre grandes villes mentionnées, très différentes les unes des autres, mais ayant en commun une population multiethnique. Il affirme que « the subalterns may not express their inner selves through speaking, but they do exactly this through their life trajectories and the choices they make along the way. Taking the idea of perceiving the body as a literary text seriously opens the way to finally hearing the subalterns speaking out $»($ p. 8).

Dans le but de comprendre « le discours du corps » (body talk), l'auteur se propose de reconstituer le langage particulier de l'autodescription et de l'autoreprésentation s'exprimant dans des pratiques sociales. Dans un style passionnant, l'auteur montre que ces villes étaient peuplées par des «Gerasim » de diverses confessions religieuses. Cet aspect complexifie l'étude de la criminalité des classes subalternes caractérisée d'une façon ethnique : le mot « classe » est pratiquement absent de cette analyse qui vise à mettre en exergue les discours concernant les groupes sociaux selon l'approche foucaldienne. L'auteur veut traquer les choix des « subalternes en tant que membres de la société plébéienne » (p. 12). Il cherche à répondre aux questions concernant les typologies de la solidarité de groupe, les formes, éventuellement cohérentes de l'économie morale ou du choix rationnel dans les différentes situations où ces individus se trouvent à agir, en approfondissant aussi la question complexe des différences culturelles et sociales.

Dans le premier chapitre, l'auteur présente son nouveau modèle « des pratiques sociales en tant que langage spécifique d'une communauté non discursive » (p. 13), car une grande quantité d'individus ordinaires, c'est-à-dire des Gerasim, s'exprimaient dans un langage du corps pouvant devenir régulier et répétitif; il montre dans quelle mesure il s'agissait de pratiques sociales qu'on peut considérer comme langage d'autoreprésentation et de communication. Parmi les habitants, il y avait des Tatars, des Juifs, des Arméniens, des Polonais, avec leurs confessions 
religieuses ; ils se côtoyaient dans les quartiers où ils travaillaient et vivaient, et l'auteur montre ce que ces différences impliquent du point de vue des pratiques sociales. Sa façon d'identifier ces pratiques sociales est aussi abordée d'un point de vue théorique, car il élabore un véritable modèle d'interprétation qui s'adapte à toutes les villes choisies. Il montre que la lecture de sources différentes (chroniques de presse ou comptes rendus de police, ainsi que lettres anonymes) offre la possibilité de réécrire l'histoire des pratiques sociales.

Le second chapitre aborde la question de la " société plébéienne » de Kazan", ville de la moyenne Volga ; il part d'une vision nouvelle de la société tsariste considérée en tant qu'espace social à plusieurs dimensions et couches sociales, dans lequel est théorisé un dispositif particulier de cohésion qui va au-delà des institutions et des autorités. L'auteur affirme en effet que « the necessity for the locals to negotiate a compromise outside any centrally imposed rules was produced there not by a deadlock of several competing empires, but by the state's (and much of patrician culture's) neglect of the everyday business of plebeian society, regardless of the ethnoconfessional status of its members » (p. 59). La narration s'attarde aussi bien sur la criminalité et les formes de violence que sur sa perception dans la presse locale au niveau des différences ethniques.

Le troisième chapitre décrit d'une façon plus approfondie les mécanismes de cohésion, en traitant le cas de la ville de Vil'na : là, on peut remarquer dans quelle mesure les différentes couches sociales s'organisaient de façon autonome dans des buts de solidarité, mais aussi à des fins de conflits (débouchant sur des pogroms). Dans la narration de l'auteur, le concept de « Patriarchality » est utile pour traquer des normes de la vie quotidienne relevant d'un ordre social supposé archaïque qui est un des aspects fondamentaux de l'agir plébéien, imperméable aux discours officiels et/ou à l'autorité. Ce concept aide, par exemple, à décrire l'attitude du chef de police Deminskij vis-à-vis de la prostitution ainsi que l'arrière-plan social de ce délit ; il met en évidence les frontières sociales de sa perception.

Le quatrième chapitre aborde la question de la violence et, en général, du comportement déviant. Il est intéressant de remarquer que « the scale of less politically motivated violence after 1905 was even greater than the outright political terrorism, although the difference between the two was quite vague » (p. 109). C'est pourquoi, le crime y est décrit du point de vue de l'anthropologie criminelle, en tant que phénomène social, c'est-à-dire dans la signification des relations sociales entourant l'individu coupable pour lequel le délit devient une rupture par rapport aux normes de l'ordre social. Il faut remarquer que l'auteur évite la notion de violence ethnique grâce à son fil rouge de " langage corporel » de violence qui révèle d'une façon indirecte aussi les divisions sociales. La partie sur la violence comme moyen de coopération est très intéressante aussi. Ainsi, la sphère de l'illégalité est un espace fondamental pour comprendre un changement social important. L'auteur a recours à Foucault ${ }^{1}$, mais on pourrait aussi évoquer certaines réflexions de Charles Tilly à propos des descriptions des changements collectifs, vus à grande échelle, à partir des individus ${ }^{2}$. 
Dans cette perspective, le cinquième chapitre aborde la question de la transformation sociale de l'expérience de l'illégalité, considérée en tant que phénomène typique des villes russes de la dernière période de la monarchie tsariste ; il s'inspire des études les plus modernes de la sociologie de la déviance, qui expliquent le crime dans sa fonction sociale aussi au niveau des différences nationales (par exemple entre Juifs et Tatars). Selon la logique de cette conception, l'auto-organisation peut déboucher sur un changement social impliquant une perception nouvelle de la déviance. Dans cette partie, l'auteur tente d'offrir une explication de l'économie morale du crime économique ainsi que les facteurs possibles pour l'étiologie de la criminalité (exploitation de la confiance d'autrui, rationalité, opportunisme et caractère ethnique). La fin du chapitre est très pointue, qui traite de l'érosion du vieil ordre par l'illégalité, car enfreindre la loi a souvent anticipé l'instauration d'un nouvel ordre.

L'auteur pense que la lecture de sources variées (chroniques de presse ou comptes rendus de police, ainsi que lettres anonymes) offre la possibilité de réécrire l'histoire des pratiques sociales. Par rapport aux études sur la criminalité, notamment celle des enfants des rues de Saint-Pétersbourg (reconstituée de façon remarquable par Joan Neuberger) ${ }^{3}$, il va au-delà du symbolisme social, car il s'interroge sur les pratiques sociales touchant la population russe subalterne. Dans l'épilogue, ce concept d'auto-organisation est mis davantage dans une perspective historique et interroge sur la signification de ce mécanisme qui place l'illégalité en avant pour expliquer la modernisation du pays et de la société.

Enfin, cet ouvrage remarquable offre un tableau inédit, très bien reconstitué de l'histoire de la violence urbaine dans quatre villes tsaristes importantes au début du vingtième siècle. C'est sans aucun doute un livre passionnant, écrit avec une grande érudition, qui marque un tournant dans sa façon d'interpréter le mécanisme social de l'auto-organisation. On ne peut pas faire grief à l'auteur de cet ouvrage, incontournable pour ceux qui s'occupent de l'histoire sociale de l'Empire tsariste, de ne pas s'être attardé sur les changements générationnels à la base d'un phénomène social visant l'inexorable rupture avec le passé.

1 - Michel Foucault, « Lives of Infamous Men » in J.D. Faubion, éd., Essential Works of Foucault, 1954-1984, vol. 3, New York : New Press, 2001, p. 157-175 ; Idem, Security, territory, Population: Lectures at the College de France (1977-1978), New York : Palgrave Mac Millan, 2009.

2 - Charles Tilly, Micro, Macro or Megrim?, in J. Schumbohm, éd., Mikrogeschichte Makrogeschichte komplementär oder inkommensurabel ? Avec les contributions de Maurizio Gribaudi, Giovanni Levi et Charles Tilly, Göttingen : Wallstein Verlag, 1998, p. 33-51.

3 -Joan Neuberger, Hooliganis : Crime, Culture, and Power in St. Petersburg, 1900-1914, Berkeley - Los Angeles - Londres : University of California Press, 1993.

\section{Dorena Caroli}

